\title{
THE POSITIVE EFFECT OF AN INTEGRATED MEDICAL RESPONSE PROTOCOL ON THE KNOWLEDGE, ATTITUDE AND PRACTICE OF MEDICAL RESPONSE DURING FLOOD DISASTER AMONG HEALTHCARE PROVIDERS IN KELANTAN: A SIMULATION-BASED RANDOMIZED CONTROLLED TRIAL
}

\author{
Mohd Faqhroll Mustaqim Mohd Fudzi ${ }^{1}$, Tuan Hairulnizam Tuan Kamauzaman ${ }^{1}$, Mohd Najib Abdul Ghani ${ }^{1}$ and \\ Hafizah Ibrahim² \\ ${ }^{1}$ Department of Emergency Medicine, School of Medical Sciences, Universiti Sains Malaysia, Kubang Kerian 16150 Kelantan, \\ Malaysia \\ ${ }^{2}$ Department of Community Medicine, School of Medical Sciences, Universiti Sains Malaysia, Kubang Kerian 16150 Kelantan, \\ Malaysia
}

Corresponding author: Tuan Hairulnizam Tuan Kamauzaman

Email: hairulnizam@usm.my

\begin{abstract}
The Integrated Medical Response protocol (IMP) is a new protocol of medical response during the response phase of a flood disaster in Kelantan, Malaysia. It integrates response workflows of various rescue agencies involved in patient care during response phase of flood disaster. Traditionally, health care services in this region used either an all-hazard protocol or those not specific to Kelantan. The present study is aimed to test the effectiveness of IMP on knowledge, attitude and practice of healthcare providers (HCP) involved in managing patients during flood disaster in Kelantan. This study was a prospective parallel group, single blinded, randomized controlled trial. The unit of randomization was the district within Kelantan on a 1:1 basis into either the control or intervention group using cluster randomized method. The hospitals within the district were subsequently assigned to the allocated group. Investigators were blinded to the assignments. The knowledge, attitude and practice scores of HCP were assessed by FloodDMQ-BM ${ }^{\odot}$ and was evaluated 2 weeks before and immediately after a flood disaster table-top exercise. Data was analyzed using two-way repeated measure ANOVA. Our findings showed that intervention was essential to improve the knowledge $[F(1,100)=6.947, p$-value $0.010(<0.05)]$ and attitude scores $[F(1,100)=31.56, p$-value 0.001]. Meanwhile, practice score was improved in both control and intervention group with time $[F(1,100)=226.56, p$-value 0.001]. Thus, our localized IMP specific to response phase of flood disaster was crucial to further enhance the knowledge and attitude levels among HCP while practice level showed similar improvement in both control and intervention group post table-top exercise.
\end{abstract}

Keywords: Flood, disasters, protocol, randomized controlled trial

\section{INTRODUCTION}

Disasters are catastrophic events that adversely affect all parts of the world. There are many types of disasters such as natural disaster for example floods, storms, drought, beach erosion, landslide and man-made disaster like explosions, fire outbreaks, pollutions, hazardous emissions ${ }^{1}$. Both natural and man-made disaster will be able to cause excess morbidity and mortality ${ }^{2}$. In 2015, the world disaster report revealed that from 2005 until 2014, flood disaster were the most frequently reported types of natural disaster worldwide at $45.9 \%$ with about 59,092 people killed and 866,417 affected $^{3}$.

The record-setting December 2014 Kelantan flood was a major flood that submerged large parts of Kelantan. The 2014 flood was the largest recorded flood in the history of Kelantan, in which 202,000 victims were displaced ${ }^{4}$. Disaster management is a multidisciplinary activity involving all departments in the hospital and must be actively involved in managing patients. Medical management during any flood disaster must be coordinated by the individuals or organizations most experienced and knowledgeable in disaster management ${ }^{5}$. Ministry of Health has published a national guideline on Flood Management in 2008. It was produced in relation with the National Security Council (NSC) Directive No $20^{6}$ in which the objective is to make sure the steps taken during flood disaster are effective and coordinated.

However, a recent local qualitative study conducted in the aftermath of the Kelantan 2014 flood highlighted the issue of insufficiency of the NSC Directive 20. It is an all-hazard disaster plan that is not specific for Kelantan. Healthcare providers (HCP) opined that they need a local, flood specific disaster plan to effectively conduct medical response in timely manner. Among the information needed in a flood disaster response protocol include local helicopter landing points, contact number of key person, flood alert system and common 
workflow across various emergency agencies. Additionally, HCPs realized that there were many disaster protocols in place by various response agencies such as police and fire bridged. These protocols varied widely in terms of definitions and workflow that affect the efficiency of medical response among various emergency services. Therefore, Integrated Medical Response protocol (IMP) has been jointly written by various relevant agencies to outline the medical response during the response phase of flood disaster in Kelantan. It streamlines response workflow of various local emergency services. This is to enable various emergency services to work in a more coordinated manner. It also contains, among others, the warning system utilized such as local river water levels and rainfall, which are not present in the NSC Directive 20. Thus, we set out to determine the effect of IMP on knowledge, attitude and practice (KAP) of medical response during the response phase of flood disaster among HCPs in Kelantan.

\section{METHODS}

\section{Study design and duration}

This study was a randomized controlled trial and conducted between December 2015 until June 2016.

\section{Participant recruitment and randomization}

Healthcare professionals of various designations from Ministry of Health hospitals and Hospital USM were recruited into the study via invitation letter. The unit of randomization was the district within Kelantan on a 1:1 basis into either the control or intervention group. The hospitals within the district were subsequently assigned to the allocated group. Cluster randomization was used to ensure administrative efficacy, lessened risk of experimental contamination and enhancement of subject compliance ${ }^{7}$. We identified eight districts to be randomized. An independent research staff, blinded as to whether they were nominating within a control or an intervention group and unaware which of their staff had consented to participate, nominated participants to this study among the hospital staff that included medical administrators, doctors, nurses or medical assistants. This trial design reduced the potential for selection bias from differential recruitment that might arise if the director of the hospital were responsible for staff selection $^{8}$. We included those involved in patient management during flood disaster in Kelantan into the study. The nominated participants were approached by researcher to explain the study and to obtain consent. They were informed that they could withdraw from the study at any time without any consequences. The allocation was concealed from all participants until the completion of preKAP measurement using FloodDMQ-BM ${ }^{\odot}$.

\section{Tools and measures}

The KAP levels of patient management during flood disaster in Kelantan were measured with the FloodDMQ- $B M^{\odot}$ questionnaire. FloodDMQ $-\mathrm{BM}^{\circ}$ is a validated questionnaire in Bahasa Malaysia language measuring knowledge, attitude and practice levels of healthcare providers managing patients during response phase of a flood disaster'. The questionnaire encompasses 4 sections: [1] demography, [2] knowledge (9 items), [3] attitude (13 items) and [4] practice (12 items). The demographic data collected include age, race, gender, place of work, duration of service and job designation. The 9 items in the knowledge section were rated on a true/false/don't know response (1 mark = correct answer, 0 mark $=$ wrong or don't know answer) with a minimum score of 0 point and a possible maximum score of 9 points. A knowledge level with a higher score indicated better participant's knowledge. Items in the attitude section were rated on a four-point scale $(1$ mark = strongly disagree, 4 marks = strongly agree). The possible minimum score of this section was 13 points and possible maximum score was 52. A higher total score in this section indicated a more positive attitude of the healthcare providers towards patient management during a flood disaster. Items in the practice section were rated on a four-point scale $(1$ mark $=$ never, 4 marks = always $)$. Total practice score assessed practice of good patient management during flood disaster. The possible minimum scoring was 12 and the possible maximum score was 48. A higher total score in this section indicates a better practice of the healthcare providers regarding patient management during a flood disaster. All items were positively structured in the attitude and practice sections therefore reverse scoring did not apply. The whole questionnaire took 10-15 minutes to complete. The internal consistency using Cronbach's alpha coefficient of attitude and practice sections tested on Kelantan healthcare providers were 0.925 and 0.935 respectively. For the dichotomous knowledge section, the marginal reliability tested on same population was acceptable at the value of $0.623^{9}$.

\section{Procedure}

All participants were required to answer the FloodDMQ-BM ${ }^{\circ}$ questionnaire (pre-KAP) after they consented to participate in the study. Participants in the control group were then given the standardized disaster response protocol while the participants in the intervention group were given the IMP protocol to be reviewed for two weeks. Subsequently, all participants were invited to a flood disaster table-top simulation where various scenarios were injected to the participants regarding patient management during the response phase of a flood disaster in Kelantan. The table-top exercise was intended to simulate a flood disaster 
affecting health services since it was not feasible to test the effect of IMP on KAP level differences after a real life-flood disaster. Participants of the tabletop simulation were arranged in groups according to their district hospitals and roles where they were expected to respond to the injected scenarios. Four conveners of the exercise were conveniently selected among Emergency Physicians expert in the field of disaster. They were prepped few days prior to the exercise to familiarize with exercise narrative. They also designed structured and standardized scenarios to inject to the participants any time they deem appropriate throughout the exercise based on participants' response. Scenarios were designed to include issues of the alert system, communication, patient triaging and transport. This was done without specific references to any disaster response protocol. The outcome of the exercise did not influence the result of the study. The whole exercise took approximately 3 hours to complete. The knowledge, attitude and practice level of patient management during a flood disaster in Kelantan among all participants were again measured after the table-top simulation (post-KAP) using FloodDMQ-BM ${ }^{\odot}$.

\section{Statistical analysis}

Continuous variables are presented as mean (standard deviation, SD), while categorical variables are presented as absolute $(\mathrm{n})$ and relative (\%) frequencies. A $p$-value of $<0.05$ was considered statistically significant. Independent t-test and paired t-test were used to analyses the parameters and displayed in profiles plots for each. Analysis was performed on intention-to-treat basis. Betweengroup analysis of variables was conducted using two-way analysis of variance (ANOVA) and reported in terms of means and standard deviations with $95 \%$ confidence intervals $(\mathrm{Cl})$. A two-way repeated measures ANOVA was conducted to determine whether there was a significant difference between control and intervention group in knowledge, attitude and practice mean scores measured at two time-points (pre-and post-KAP) as well as the resulting two-factor interaction of group and time. Model assumptions of normality, homogeneity of variance and compound symmetry were checked.
Significance level was set at 0.05 . The analyses were performed using SPSS version 22.0.

\section{Ethical issues}

Ethical approval was obtained from Human Research Ethics Committee Universiti Sains Malaysia (USM/JEPeM/15100333). The study was also approved by Medical Research and Ethical Committee, Ministry of Health Malaysia (NMRR-151700-27385-IIR). There was no conflict of interest among the researchers.

\section{RESULTS}

Eight district hospitals were randomized. Out of 132 candidates, a total of 120 were eligible to participate in this study. Sixty candidates were allocated in each group of control and intervention. However, 13 were lost to follow-up and 5 had missing data and were not included in the analysis. Therefore, only 102 respondents were included in the final analysis giving a response rate of $85.0 \%$ $(102 / 120)$. The CONSORT diagram is shown in Figure 1.

\section{Demography of the respondents}

The mean (SD) for age of respondents and period of service were 39.8 (7.5) and 14.1 (7.2) respectively. Majority of the respondents were Malay $(96.1 \%)$, male $(60.8 \%)$ and worked in district hospital $(41.2 \%)$. Most of the respondents had prior experience in managing disaster at the scene (55.9\%) and being victim in a disaster themselves but not in the hospital-setting $(57.8 \%)$. Most of them $(86.3 \%)$ never had any training in flood disaster before (Table 1).

The demographic data of the two groups are summarized in Table 1. There were no significant differences in age, period of services, race, sex, place of service, job, experience in managing patient at scene or hospital, history of being victim or prior disaster training between the groups. Therefore, assumption of homogeneity between the control and intervention group can be made (Table 1). 


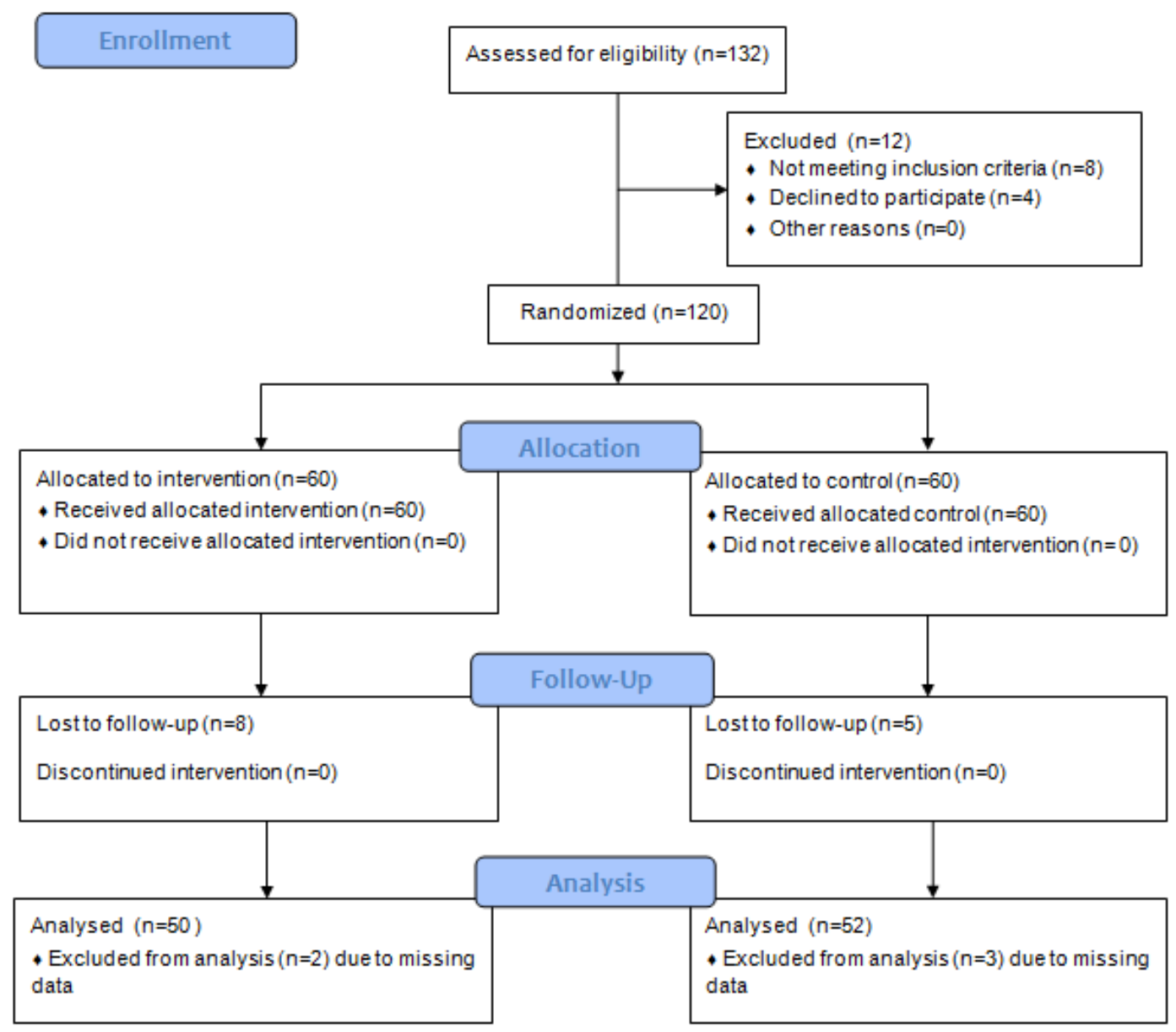

Figure 1: CONSORT flow diagram

\section{Impact of the IMP on knowledge}

At baseline (pre-intervention), the mean knowledge score between the control and intervention are 3.31 \pm 1.51 vs. $2.98 \pm 1.50$. The level of knowledge improved significantly for both the control and intervention group over time (Figure 2). Within the control group, the mean knowledge score improved with time $(5.40 \pm 2.11)$ whereby in the intervention group increase to greater extent $(6.56 \pm 1.69)$. This showed that the knowledge level improved by 72.5 $\%$ with time but further increase to $120 \%$ after intervention. Therefore, there was overall mean difference on knowledge score whereby the mean score of the intervention group post intervention was significantly higher as compared to the control group $[F(1,100)=6.947, p$-value $0.010(<0.05)]$ (Table 2).

\section{Impact of the IMP on attitude}

At baseline, the mean attitude score in control and intervention group are $46.29 \pm 4.62$ and $48.26 \pm 4.28$, respectively. The attitude score did not show much improvement in control group $(46.33 \pm 4.82)$ with time. However, we observed the intervention group showed better improvement $(50.40 \pm 1.28)$ after intervention (Figure 3 ). This showed that contrary to the control group, the attitude scores improved after intervention by $4.4 \%$ increment. Hence, there is no significant change of attitude scores over time but there was an overall mean difference after intervention on attitude score $[\mathrm{F}(1,100)=31.56, p$ value 0.001] (Table 2). 


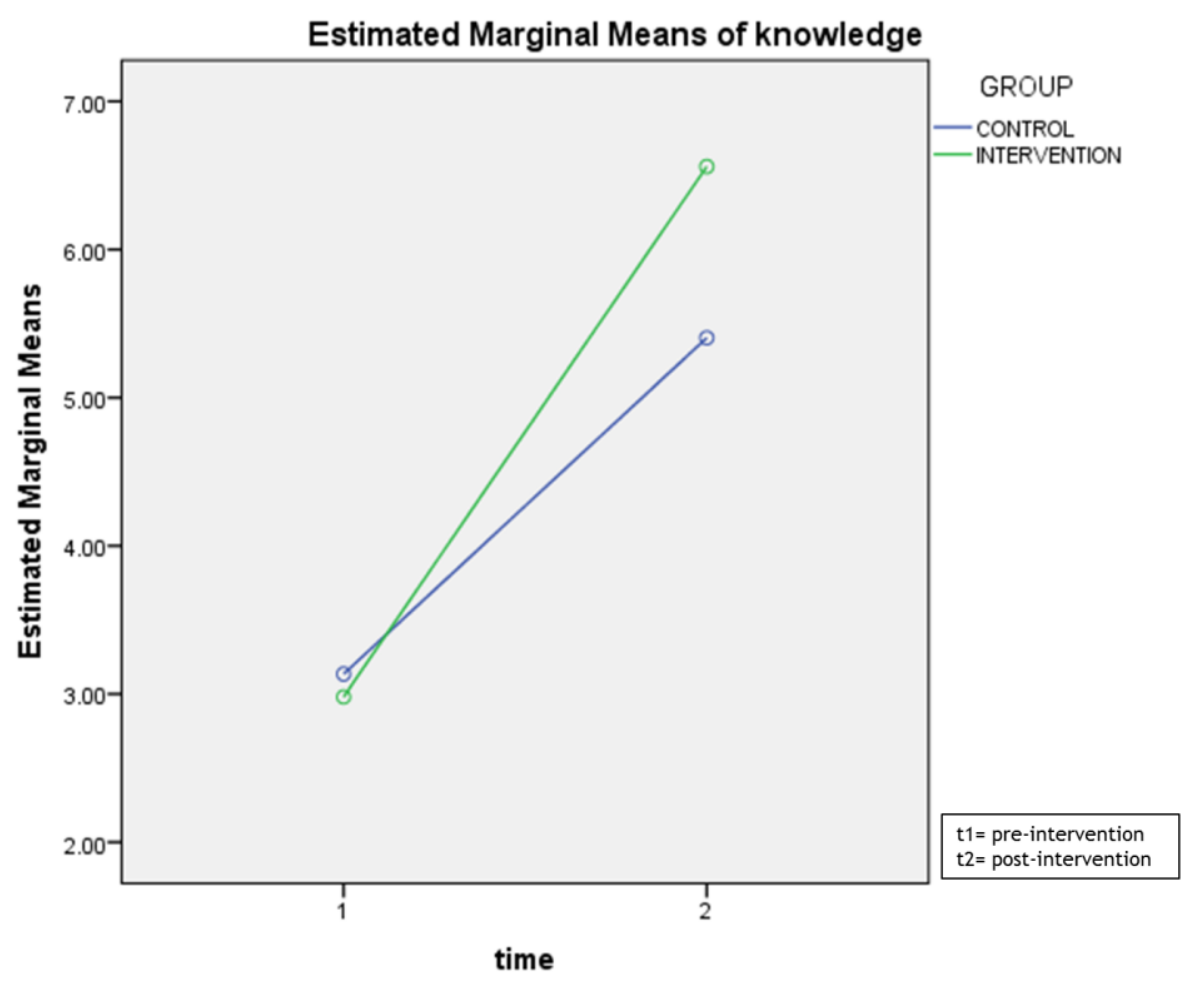

Figure 2: Profile plot of changes in knowledge score between control and intervention over time.

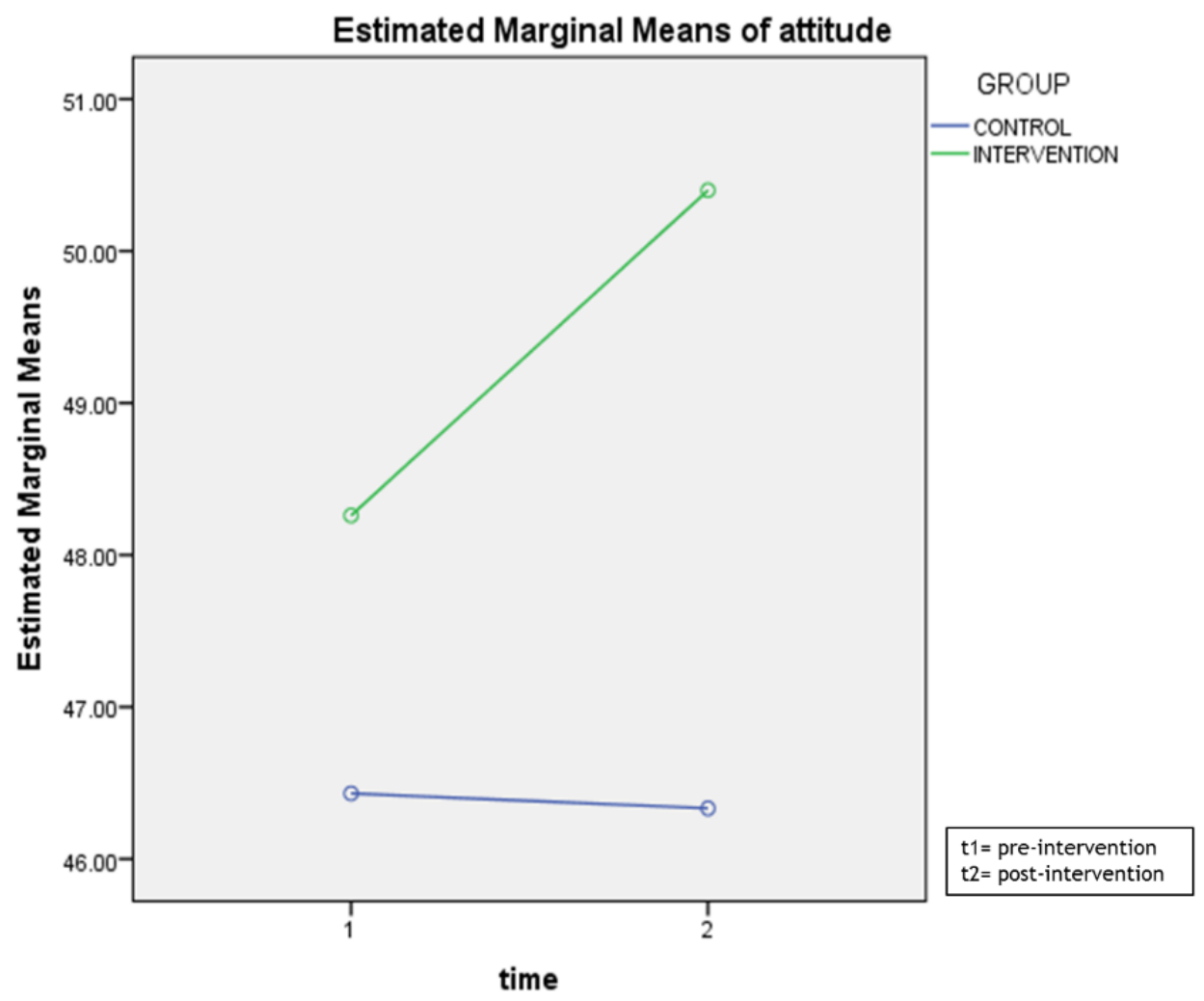

Figure 3: Profile plot of changes in attitude score between control and intervention over time. 
Impact of IMP on practice

With regards to the practice score, the mean practice score at baseline for control group was $31.13 \pm 6.62$ and intervention group was $31.54 \pm 9.93$. Both the control and intervention group showed significant improvement in the practice score with time but not after intervention. We observed that the mean practice score improved with time for both control and intervention group ( $44.69 \pm 3.91$ vs $45.52 \pm 3.12$ ) (Figure 4). Thus, there is no significant change of practice scores after intervention but there was an overall mean difference with time on practice score $[F(1,100)=226.56, p$-value 0.001$]$ (Table 2)

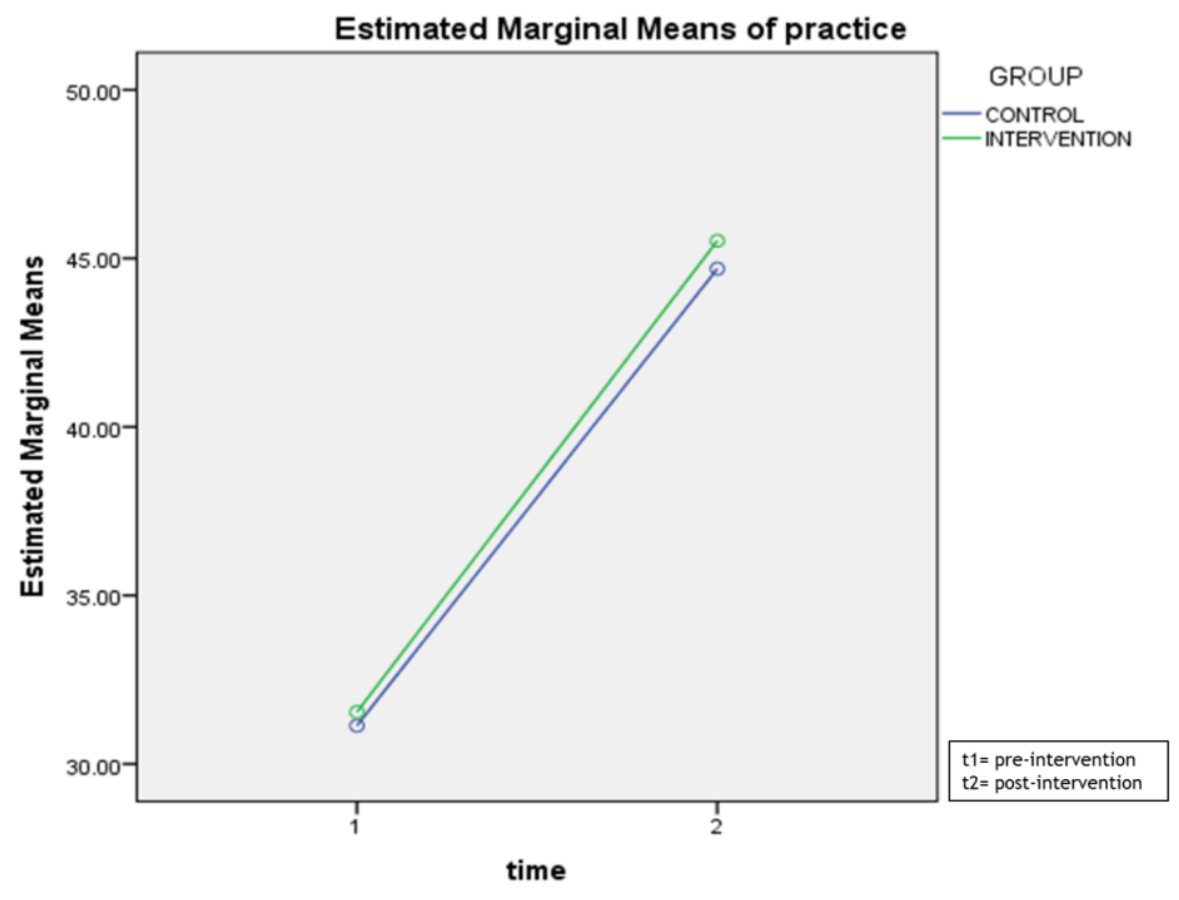

Figure 4: Profile plot of changes in practice score between control and intervention over time.

\section{DISCUSSION}

To the best of our knowledge, this is the first Malaysia-based cluster randomized controlled trial, to investigate the effectiveness of a disaster protocol on the knowledge, attitude and practice of medical response among HCP involved in patient management during flood disaster. The strength of this study lies within its design of prospective randomized study using a validated flood disaster preparedness questionnaire.

Given the scarcity and unpredictability of major flood disaster ocurance in Kelantan, it was difficult to investigate the effectiveness of IMP during a real flood disaster. Therefore, we studied the effects over a flood disaster table-top simulation, a method that simulates real-life disaster ${ }^{10}$. Simulation-based exercise is nothing new within emergency and disaster preparedness and has been increasingly used to achieve the required knowledge, skills and experienced ${ }^{11}$. Furthermore, table-top exercise provided additional benefits ${ }^{12}$ for some limitations of field operation drills such as communication, coordination and post event mitigation ${ }^{13,14}$. It is a formative assessment strategy that identifies lessons learned regarding strengths and challenges for policies, procedures and assists in transferring passive learning into experiences that mimic real life ${ }^{15,16}$. However, in our study the exercise assessment were not done since it will not affect the study results. To date, there are not many research investigating effects of any interventions to improve disaster management globally, mostly due to the inherent difficulties faced by research on natural disaster ${ }^{17}$.

Most of the literatures regarding disaster management is based on research reporting "lessons learned" rather than objective measures ${ }^{18,19}$. Additionally, a recent scoping review in 2017 showed that most studies reported only to the extent of identifying gaps in managing disaster and sharing the lessons from emergency exercises ${ }^{15}$. In contrast, the finding from our study added to the understanding of how a medical response protocol that is locally and flood specific as well as streamlined across various agencies, can have a positive effect on the knowledge, attitude and practice level. 
Table 1: Distribution of sociodemographic characteristics of respondents for the intervention and control groups.

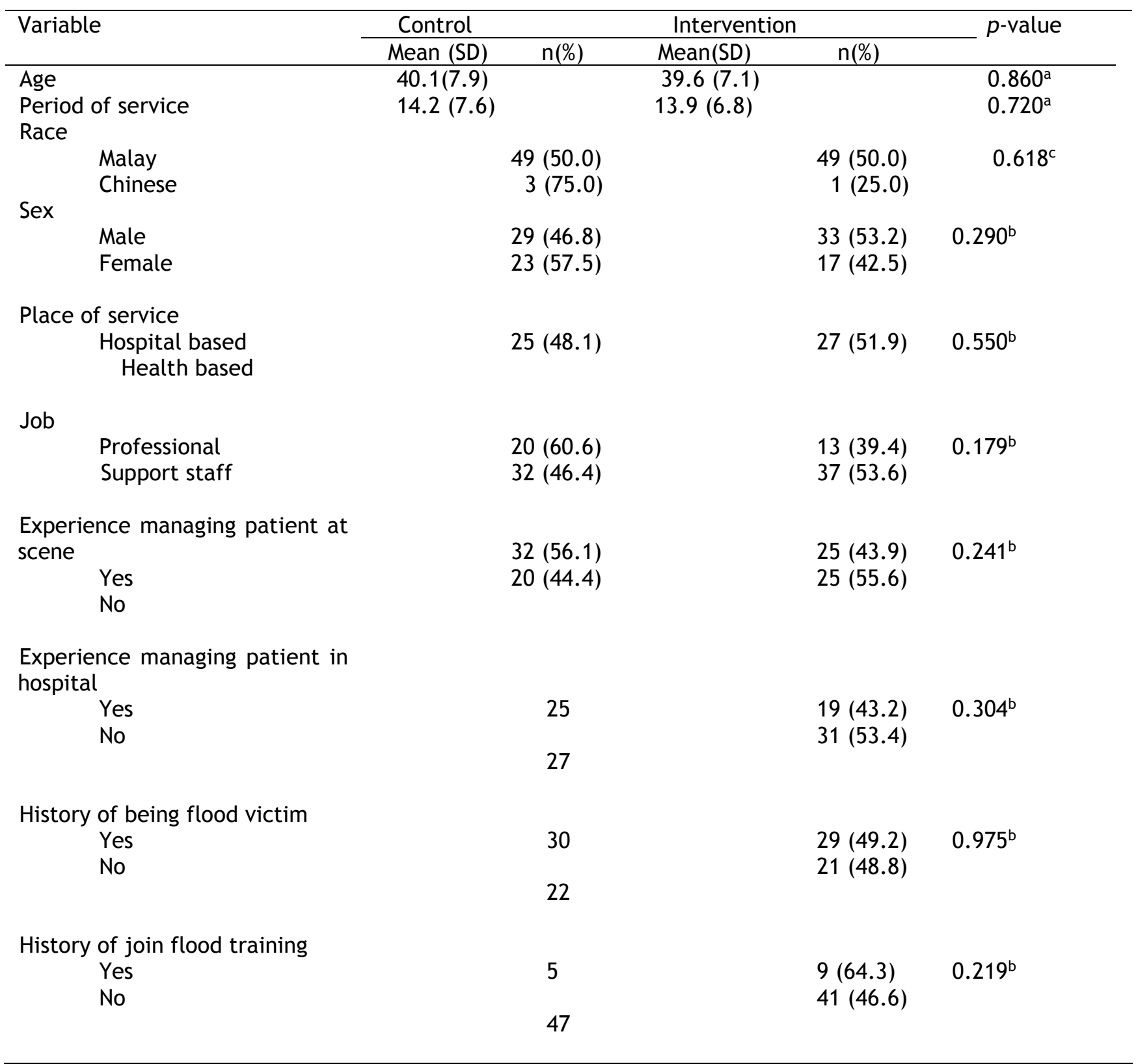

andependent t test. 'PPearson's chi-Square test. 'Fisher's exact test.

HCP in Malaysia relied mainly on the flood disaster protocol and NSC Directive 20 provided by the Ministry of Health, Malaysia published in 2008. According to Linkous and Carter, regional planning at local levels is the key to successful response during most disasters ${ }^{20}$. Therefore, the IMP was locally designed from a collaboration between agencies during flood disaster management ${ }^{21}$. The importance of refining emergency protocol through exercises to produce a workable document was demonstrated by the success in evacuation, transport and placement of patients during interfacility drills as reported by Verni in $2012^{22}$.
We found that the level of knowledge among HCPs improved with time and was significantly increased to a greater extent in the intervention group. A positive change in the knowledge level would indicate that an intervention is effective and indicates that learning has taken place ${ }^{23}$. Similarly, few studies reported that the knowledge level significantly improved with intervention and time ${ }^{23,24}$. Among the knowledge related to field of disaster medicine that need to be emphasized are communication and coordination between centers and agencies, medical surge capacity, staff deployment, and patient management ${ }^{15}$. In addition, all HCP must be trained and equipped with specific knowledge and competencies, for example 
understanding the importance of triaging patient during disaster ${ }^{25}$. However, an increase in knowledge level score not necessarily correlates with improvement in respond to an actual disaster ${ }^{18}$. This is reflected by our result that shows no significant change of practice level with IMP although significant improvement with time (after table-top exercise) was observed. We theorized that while knowledge is based on factual information and theoretical concepts that can be acquired by reading the IMP, practice level might require repeated experience (actual disaster or simulation exercise) to have significant change.

Table 2: Results of two-way repeated measures ANOVA for knowledge, attitude and practice

\begin{tabular}{|c|c|c|c|c|c|c|c|c|c|}
\hline \multirow[t]{2}{*}{ Parameters } & \multirow[t]{2}{*}{ Group } & \multirow[t]{2}{*}{ Time } & \multirow[t]{2}{*}{$\begin{array}{l}\text { Mean } \\
(\text { SD) }\end{array}$} & \multicolumn{2}{|c|}{ Effect of time } & \multicolumn{2}{|c|}{$\begin{array}{l}\text { Effect of time } x \\
\text { group }\end{array}$} & \multicolumn{2}{|c|}{ Effect of group } \\
\hline & & & & $\begin{array}{l}F \\
(1,100)\end{array}$ & $\begin{array}{l}p- \\
\text { value }\end{array}$ & $\begin{array}{l}F \\
(1,100)\end{array}$ & $\begin{array}{l}p- \\
\text { value }\end{array}$ & $\begin{array}{l}F \\
(1,100)\end{array}$ & $p$-value \\
\hline \multirow[t]{4}{*}{ Knowledge } & Control & Pre & $\begin{array}{l}3.13 \\
(1.51)\end{array}$ & 138.34 & $<0.001$ & 6.947 & 0.010 & 4.59 & 0.035 \\
\hline & Intervention & & $\begin{array}{l}2.98 \\
(1.50)\end{array}$ & & & & & & \\
\hline & Control & Post & $\begin{array}{l}5.40 \\
(2.11)\end{array}$ & & & & & & \\
\hline & Intervention & & $\begin{array}{l}6.56 \\
(1.69)\end{array}$ & & & & & & \\
\hline \multirow[t]{4}{*}{ Attitude } & Control & Pre & $\begin{array}{l}46.43 \\
(4.55)\end{array}$ & 2.90 & 0.092 & 3.487 & 0.065 & 31.56 & $<0.001$ \\
\hline & Intervention & & $\begin{array}{l}48.26 \\
(4.28)\end{array}$ & & & & & & \\
\hline & Control & Post & $\begin{array}{l}46.33 \\
(4.82)\end{array}$ & & & & & & \\
\hline & Intervention & & $\begin{array}{l}50.40 \\
(1.28)\end{array}$ & & & & & & \\
\hline \multirow[t]{4}{*}{ Practice } & & Pre & $\begin{array}{l}31.13 \\
(6.62)\end{array}$ & 226.56 & $<0.001$ & 0.053 & 0.818 & 0.478 & 0.491 \\
\hline & Intervention & & $\begin{array}{l}31.54 \\
(9.93)\end{array}$ & & & & & & \\
\hline & Control & Post & $\begin{array}{l}44.69 \\
(3.91)\end{array}$ & & & & & & \\
\hline & Intervention & & $\begin{array}{l}45.52 \\
(3.12)\end{array}$ & & & & & & \\
\hline
\end{tabular}

Contrary to the knowledge level observation, a positive improvement in the attitude level among HCP was observed after participants were provided with IMP as compared to the control but not affected by the table-top experience. We postulate that this might stem from the Hawthorn effect where the participants might modify their attitude when knowing they are being experimented. This might be especially true when responding on a Likert-scale to statements in the attitude section such as: "I need to refer to the response protocol when working during a flood disaster", "I need to know about the patient transportation process during a flood disaster" and "I need to monitor the river level during a flood disaster". Respondents in the intervention group might have a better awareness of communication, patient transport process, alert system and function of other agencies since these are outlined in the IMP. This might translate into a better attitude during a flood disaster and will be observed regardless of whether they go through the disaster experience or not. However, improvement in attitude in terms of team capabilities, personal abilities, and teamwork associated with emergency response has been reported with interventions to improve emergency response ${ }^{23,26}$. Additionally, it can be argued that measuring attitude scores immediately postexercise are imperfect indicators of learning, as they may indicate only temporary change rather than lasting learning ${ }^{27}$. 
Finally, our results demonstrated that the level of practice did not improve significantly with IMP but affected by experience (table-top simulation). In general, positive improvement in practice level was observed over time and experience ${ }^{10,15}$. The pervasiveness of poor staff emergency preparedness and training and reported difficulties in attempting to educate staff in a hospital disaster plan may explain why the intervention was not crucial in improving level of practice in our study ${ }^{29-31}$. "Lack of interest and other priorities" may contribute to the insignificant change in our study, comparing both control and intervention groups. Ford and Schmidt reported that among the practice that found to be important were how to activate disaster plans, the role of medical staff and how to participate in a coordinated response ${ }^{27}$. However, improved knowledge may not predict improved performance in real disaster response because of the many challenges of translating knowledge into practical competence ${ }^{32}$. We are of the opinion that in order to have a significant change in practice of responding to flood disaster, the HCP has to experience several real-life disasters using the IMP. The limitation of table-top simulation used in the study was that it only enable the participant to exercise decision makings. The effect on practice needs to be further tested on real disasters. Although the IMP has added few important elements such as local flood alert denominators, helicopter landing points and important phone numbers to make it functional during disaster, improvements need to be made to make it practically functional. It needs to be tested and reviewed constantly.

\section{Limitations}

Our findings should be interpreted with several limitations in mind. Due to the periodicity of measurement whereby only a single measurement post intervention was taken, a true longitudinal effect of IMP is difficult to determine. The result of this study also may be influenced by other factors such as exposure to other media and contamination of samples between control and intervention group. This study did not measure the knowledge, attitude and practice level in regard to different sociodemographic distribution for example type of job, work place and working experience. Furthermore, evaluating the effects of disaster training is however complicated as real skill can only be demonstrated during a real major incident $^{16}$. To ensure that there was no contamination of control and intervention group, measures were taken which included randomization of districts rather than the subjects per se.

\section{Recommendations}

We would like to recommend regular and proper training to create a readiness for managing the impacts of disaster. Emphasizing the importance of pre-exercise preparation and addressing the principles of adult learning, as an educational opportunity, may improve the outcomes and contribute to effective response in real life event. It requires augmentation of the training facilities and the provision of opportunities by the health care authorities to enable medical personnel to fully participate. Additionally, future research should incorporate large scale of various types of HCP to be carried out during actual flood disaster and need to be evaluated and reported. Furthermore, we would like to recommend each locality to produce their own flood disaster protocol to ensure successful response during disaster.

\section{CONCLUSIONS}

The present study was the first ever study conducted as a prospective randomized study with regards to the field of disaster management in Malaysia. Based on our findings, we can conclude that our locally designed flood disaster management protocol (IMP) contribute significantly to the improvement of knowledge and attitude scores among HCP. Contrary to knowledge and attitude scores, intervention has no significant outcomes on practice score. Therefore, the IMP has a very large potential for the future flood disaster management as showed in our study.

\section{ACKNOWLEDGEMENT}

Our special thanks to the School of Medical Sciences, Universiti Sains Malaysia for supporting this study. Our appreciation to all the staffs in Hospital USM involved in this study and their help. This study was funded by Ministry of Education Malaysia Trans-Disiplinary Research Grant Scheme (TRGS). The FloodDMQ-BM is copyrighted to Universiti Sains Malaysia.

\section{REFERENCES}

1. Chan NW. Flood disaster management in Malaysia: an evaluation of the effectiveness of government resettlement schemes. Disaster Prevention and Management: An International Journal. 1995;4(4):22-9.

2. Persson ES, Nyberg L, Svedung I. Flood warning in a Swedish local risk management context. Disaster Prevention and Management: An International Journal. 2015;24(3):383-96.

3. Rattanakanlaya K, Sukonthasarn A, Wangsrikhun S, Chanprasit C. A survey of flood disaster preparedness among hospitals in the central region of Thailand. 
Australasian Emergency Nursing Journal. 2016;19(4):191-7.

4. Su-Lyn B. Kelantan floods like Japan's 2011 Tsunami, says NSC. 2015. Malaysia: The Malay Mail Online; 2015.

5. Franc JM, Nichols D, Dong SL. Increasing Emergency Medicine Residents' Confidence in Disaster Management: Use of an Emergency Department Simulator and an Expedited Curriculum. Prehospital and Disaster Medicine. 2012;27(01):31-5.

6. Agensi Pengurusan Bencana (NADMA). Arahan MKN No. 20 : Dasar dan Mekanisme Pengurusan Bencana Negara 2012 [cited 2019 5/1/2019]. Available from: http://portalbencana.nadma.gov.my.

7. Donner A, Klar N. Pitfalls of and Controversies in Cluster Randomization Trials. American Journal of Public Health. 2004;94(3):416-22.

8. Farrin A, Russell I, Torgerson D, Underwood M. Differential recruitment in a cluster randomized trial in primary care: the experience of the UK Back pain, Exercise, Active management and Manipulation (UK BEAM) feasibility study. Clinical Trials. 2005;2(2):119-24.

9. Ghani A, Tuan Hairulnizam TK, MFM MF, Arifin WN, Chew KS. Development and psychometric evaluation of flood disaster management questionnaire-(FloodDMQ$\mathrm{BM})$ : Exploratory factor analysis and item response theory analysis. International Journal of Public Health and Clinical Sciences. 2016 Jun 21;3(3):59-70.

10. Kaplan BG, Connor A, Ferranti EP, Holmes $L$, Spencer L. Use of an emergency preparedness disaster simulation with undergraduate nursing students. Public health nursing (Boston, Mass). 2012;29(1):44-51.

11. Jonson C-O, Pettersson J, Rybing J, Nilsson $H$, Prytz E. Short simulation exercises to improve emergency department nurses' self-efficacy for initial disaster management: Controlled before and after study. Nurse Education Today. 2017;55:205.

12. Savoia E, Biddinger PD, Fox P, Levin DE, Stone L, Stoto MA. Impact of Tabletop Exercises on Participants' Knowledge of and Confidence in Legal Authorities for
Infectious Disease Emergencies. Disaster Med Public Health Prep. 2013;3(2):104-10.

13. Handbook on simualation exercises in EU public health setting - How to develop simulation exercises within the framework of public health response to communicable diseases. [Internet]. ECDC. 2014.

14. Chi C-H, Chao W-H, Chuang C-C, Tsai M-C, Tsai L-M. Emergency medical technicians' disaster training by tabletop exercise. The American Journal of Emergency Medicine. 2001;19(5):433-6.

15. Skryabina E, Reedy G, Amlôt R, Jaye P, Riley $P$. What is the value of health emergency preparedness exercises? A scoping review study. International Journal of Disaster Risk Reduction. 2017;21:274-83.

16. Barton A. Patient Safety and Quality: An Evidence-Based Handbook for Nurses. AORN Journal.90(4):601-2.

17. Nazli NNNN, Sipon S, Radzi HM. Analysis of Training Needs in Disaster Preparedness. Procedia - Social and Behavioral Sciences. 2014;140:576-80.

18. Williams J, Nocera M, Casteel C. The Effectiveness of Disaster Training for Health Care Workers: A Systematic Review. Annals of Emergency Medicine. 2008;52(3):21122.e2.

19. Hsu EB, Jenckes MW, Catlett CL, Robinson $\mathrm{KA}$, Feuerstein $\mathrm{C}$, Cosgrove SE, et al. Effectiveness of Hospital Staff MassCasualty Incident Training Methods: A Systematic Literature Review. Prehospital and Disaster Medicine. 2012;19(3):191-9.

20. Linkous D, Carter KF. Responding to the Shootings at Virginia Tech: Planning and Preparedness. Journal of Emergency Nursing. 2009;35(4):321-5.

21. Tuan Hairulnizam TK, Rahman NHNA, Sheng CK, Fudzi MFMM, Hamzah MSSC, Ghani MNA, et al. Results of Focus Group Discussion and Elite Interview on Patient Managment during Flood Disaster in Kelantan. Kelantan International Health Conference: School of Medicine, Universiti Sains Malaysia; 2015.

22. A Hospital System's Response To A Hurricane Offers Lessons, Including The Need For Mandatory Interfacility Drills. Health Aff (Millwood). 2012;31(8):1814-21. 
23. M. PD, W. PR. The impacts of disaster exercises on participants. Disaster Prevention and Management: An International Journal. 1999;8(4):241-55.

24. Klima DA, Seiler SH, Peterson JB, Christmas $A B$, Green JM, Fleming $G$, et al. Full-scale regional exercises: closing the gaps in disaster preparedness. The journal of trauma and acute care surgery. 2012;73(3):592-7; discussion 7-8.

25. McBrien B. Translating change: The development of a person-centred triage training programme for emergency nurses. International Emergency Nursing. 2009;17(1):31-7.

26. Perry RW. Disaster Exercise Outcomes for Professional Emergency Personnel and Citizen Volunteers. Journal of Contingencies and Crisis Management. 2004;12(2):64-75.

27. Ford JK, Schmidt AM. Emergency response training: strategies for enhancing realworld performance. Journal of Hazardous Materials. 2000;75(2-3):195-215.

28. Sarpy SA, Warren CR, Kaplan S, Bradley J, Howe R. Simulating public health response to a severe acute respiratory syndrome (SARS) event: a comprehensive and systematic approach to designing, implementing, and evaluating a tabletop exercise. Journal of public health management and practice. 2005;11(6):S75S82.

29. Leiba A, Goldberg A, Hourvitz A, Weiss G, Peres $M$, Karskass $A$, et al. Who Should Worry for the "Worried Well"? Analysis of Mild Casualties Center Drills in NonConventional Scenarios. Prehospital and Disaster Medicine. 2012;21(6):441-4.

30. Vinson E. Managing Bioterrorism Mass Casualties in an Emergency Department: Lessons Learned From a Rural Community Hospital Disaster Drill. Disaster Manag Response. 2007;5(1):18-21.

31. Cone DC, MacMillan DS, Parwani V, Van Gelder C. Pilot Test of a Proposed Chemical/Biological/Radiation/ NuclearCapable Mass Casualty Triage System. Prehosp Emerg Care. 2008;12(2):236-40.

32. Beaton RD, Johnson LC. Instrument Development and Evaluation of Domestic Preparedness Training for First Responders.
Prehospital and Disaster Medicine. 2012;17(3):119-25. 